06

\title{
Нелинейные акустические эффекты в поликристаллических твердых телах с дислокациями
}

\author{
(C) B.E. Назаров
}

Институт прикладной фризики РАН, 603950 Нижний Новгород, Россия

e-mail: v.e.nazarov@appl.sci-nnov.ru

Поступило в Редакцию 16 января 2020 г.

В окончательной редакции 2 июня 2020 г.

Принято к публикации 6 июня 2020 г.

В рамках модифицированной линейной части дислокационной теории поглощения Гранато-Люкке получено уравнение состояния для поликристаллических твердых тел с дислокационной диссипативной и реактивной акустической нелинейностью. Проведено теоретическое исследование нелинейных эффектов, связанных с влиянием статической нагрузки и мощной низкочастотной стоячей упругой волны в стержневом резонаторе на распространение слабой (пробной) волны, а также эффектов самовоздействия интенсивной бегущей волны и генерации ее третьей гармоники.

Ключевые слова: поликристаллы, дислокационная теория поглощения, диссипативная и реактивная нелинейности, упругие волны.

DOI: $10.21883 / J T F .2020 .12 .50126 .17-20$

\section{Введение}

Акустические свойства поликристаллических твердых тел, в частности, металлов и горных пород, во многом определяются дислокациями - одномерными (или линейными - в геометрическом смысле) дефектами их кристаллической решетки. Дислокации - это линии, вдоль (и вблизи) которых нарушено правильное расположение атомных плоскостей кристалла. Дислокации образуются при кристаллизации, в процессе роста поликристалла и при его пластической деформации (наклепе) [1-6]. Для металлов плотность дислокаций изменяется при термической обработке (отжиге) и при наклепе, причем при отжиге плотность дислокаций падает, а при наклепе - растет [1-6].

Существуют два основных вида дислокаций: краевые и винтовые. Краевая дислокация - это граница неполной атомной плоскости (экстраплоскости) в кристаллической решетке кристалла. Винтовая дислокация такой экстраплоскости не содержит, при этом все атомы кристалла находятся на одной винтовой поверхности, закрученной вокруг дислокационной линии (оси дислокации). В реальных поликристаллах дислокации являются смешанными; они представляют собой сочетание основных видов дислокаций и содержат как краевую, так и винтовую составляющие. Имеют место два разных типа движения дислокаций: консервативное (или скольжение) и неконсервативное (или переползание) [1-6].

Консервативное движение происходит в плоскости скольжения дислокации под действием относительно малых сдвиговых напряжений. Скольжение представляет собой перераспределение (т.е. разрыв старых и образование новых) атомных связей вблизи линии дислокации. Консервативное движение дислокаций не связано с перемещением атомов вещества, оно практически не зависит от температуры. Скольжение могут совершать как краевые, так и винтовые дислокации [1-6].

Неконсервативное движение, наоборот, происходит в направлении, перпендикулярном плоскости скольжения дислокации, под действием относительно больших продольных напряжений. Неконсервативное движение связано с перемещением атомов вещества вблизи линии дислокации. В зависимости от знака напряжения (т.е. от растяжения или сжатия) атомы кристалла перемещаются к линии дислокации или от нее. Переползание дислокаций - это диффузионный, термически активируемый процесс, его скорость растет с ростом температуры. При небольших напряжениях, характерных для акустических волн, и не очень высокой температуре переползание дислокаций маловероятно. Переползание могут совершать только краевые дислокации [1-6].

Важным и отличительным свойством дислокаций является их высокая подвижность (под действием внешнего напряжения) и сильное упругое взаимодействие между собой и точечными дефектами решетки (вакансиями, межузельными и примесными атомами), что существенно влияет на механические и акустические (упругие и неупругие, линейные и нелинейные) свойства поликристаллов. Поле упругих напряжений для дислокаций уменьшается как $s^{-1}$, а для точечных дефектов как $s^{-3}$, где $s-$ расстояние от ядра дислокации или от точечного дефекта до точки наблюдения. Вынужденные под действием слабых высокочастотных (ВЧ) напряжений колебания дислокаций являются причиной линейного резонансного внутреннего трения - амплитуднонезависимых поглощения и дисперсии ВЧ акустических волн в поликристаллах [7-14]. При относительно сильных низкочастотных (НЧ) напряжениях колебания 
дислокаций и их взаимодействие с примесными атомами приводит к гистерезисной нелинейности поликристаллов и к проявлению в таких средах эффектов амплитуднозависимого внутреннего трения (АЗВТ) [7-14], генерации высших гармоник и других нелинейных акустических эффектов (НАЭ).

В разных поликристаллических твердых телах НАЭ проявляются по-разному, что свидетельствует о различной нелинейной динамике дислокаций, зависящей от большого числа структурных характеристик поликристалла (типа решетки, функции распределения, плотности и вида дислокаций, концентрации вакансий, межузельных и примесных атомов, размеров зерна и т.д.). Нелинейные акустические (упругие и неупругие) свойства поликристаллов более чувствительны к их дислокационной структуре, чем линейные, поэтому исследования нелинейных волновых процессов в таких средах способствуют изучению динамики дислокаций и созданию моделей их движения под действием динамических напряжений, что необходимо для развития теории прочности и пластичности - одного из актуальных направлений физики твердого тела [1-14].

Для объяснения явления внутреннего трения поликристаллических металлов Гранато и Люкке на основе струнной модели дислокации Келера [15] создали дислокационную теорию поглощения [7-14]. В этой теории полагается, что под действием переменного напряжения упругой волны дислокации, жестко закрепленные в узлах сетки - в точках пересечения дислокаций совершают вынужденные колебания консервативного типа. Дислокационная теория Гранато-Люкке состоит из двух частей - ВЧ линейной и НЧ гистерезисной (нелинейной), и определяет соответственно линейные (амплитудно-независимые) и нелинейные (амплитуднозависимые) потери и дефект модуля упругости. Линейные потери и дефект модуля упругости имеют место при малых динамических напряжениях, недостаточных для отрыва сегментов дислокаций от примесных атомов; они связаны с торможением движущихся дислокаций в вязкой среде, зависят от частоты и проявляются на высоких частотах - в области резонансных частот дислокаций (в диапазоне десятков и сотен мегагерц). Нелинейные потери и дефект модуля упругости наблюдаются при относительно больших напряжениях на относительно низких частотах. Они связаны с отрывом сегментов дислокаций от примесных атомов и с различным их поведением на стадиях нагрузки и разгрузки; это определяет гистерезисный характер уравнения состояния поликристалла. В теории Гранато-Люкке гистерезисная нелинейность безынерционна, и эффекты АЗВТ от частоты волны не зависят. В действительности же с ростом частоты деформирования гистерезисная нелинейность поликристаллов уменьшается [16].

Теория Гранато-Люкке вполне удовлетворительно описывает эффекты линейного внутреннего трения и качественно объясняет результаты измерений амплитуднозависимых декремента затухания и дефекта модуля упругости во многих достаточно чистых металлах (в НЧ диапазоне). Для металлов же с высокой концентрацией примесей результаты измерений часто не соответствуют теории, при этом могут наблюдаться различные амплитудные зависимости гистерезисных эффектов для, казалось бы, одного и того же металла [17-19], что связано с большой чувствительностью АЗВТ к малому изменению концентрации примесей и плотности дислокаций. Кроме того, в некоторых поликристаллических металлах и горных породах (отожженная медь, цинк, свинец, гранит, мрамор, песчаник, известняк, магнезит, кварцит и т.д.) наблюдаются также и другие чрезвычайно сильные нелинейные эффекты, в частности, затухание и фазовая задержка несущей слабой ВЧ волны под действием сильной НЧ волны накачки, не описываемые теорией Гранато-Люкке и обусловленные не НЧ гистерезисной, а ВЧ диссипативной (неупругой) и реактивной (упругой) нелинейностью [20-30]. Изучение НАЭ в поликристаллических твердых телах можно использовать для развития дислокационной теории поглощения и уточнения (или модификации) уравнения движения дислокации - основного дефекта поликристалла, ответственного за его физические свойства: упругость, вязкость, пластичность, прочность, акустическую нелинейность и т.д.

В настоящей работе проводится теоретическое исследование эффектов влияния статической нагрузки и мощной НЧ стоячей упругой волны на распространение слабой (пробной) ВЧ волны в поликристаллах, обладающих дислокационной диссипативной и реактивной акустической нелинейностью, а также эффектов самовоздействия интенсивной бегущей волны и генерации ее третьей гармоники. Описание этих эффектов проводится в рамках нелинейно-модифицированной линейной ВЧ части дислокационной теории Гранато-Люкке. При получении уравнения состояния поликристалла с дислокациями мы не будем учитывать линейную диссипацию однородного твердого тела (не содержащего дислокаций) и его слабую решеточную упругую нелинейность, описываемую пятиконстантной теорией упругости [31].

\section{1. Основные уравнения для поликристаллических твердых тел с диссипативной и реактивной дислокационной нелинейностью}

Для описания нелинейных акустических эффектов, в частности, затухания и фазовой задержки несущей слабой ВЧ волны под действием сильной НЧ волны накачки, не связанных с НЧ гистерезисной нелинейностью, модифицируем ВЧ линейную часть дислокационной теории Гранато-Люкке [7-10] и получим нелинейное уравнение состояния поликристалла. Для этого смещение $\xi=\xi(y, t)$ сегмента дислокации длины $l$ под действием переменного сдвигового напряжения $\tau=\tau(t)$ 
будем также описывать уравнением колебаний струны, но содержащим малые нелинейные диссипативное (неупругое) и реактивное (упругое) слагаемые, учитывающие нелинейные трение и натяжение дислокации:

$$
\begin{aligned}
& A \xi_{t t}+B\left[1+\mu\left(|\xi / b|^{m-q}\left|\xi_{t} / C_{\perp}\right|^{q}\right)\right] \xi_{t} \\
& -C\left[1-\eta\left(|\xi / b|^{n-r}\left|\xi_{t} / C_{\perp}\right|^{r}\right)\right] \xi_{y y}=b \tau(t),
\end{aligned}
$$

где $A=\pi \rho b^{2}$ - масса единицы длины дислокации; $B-$ коэффициент линейного трения $(B>0)$; $C=2 G b^{2} / \pi(1-v)-$ коэффициент линейного натяжения; $G, v, \rho$ и $C_{\perp}=(G / \rho)^{1 / 2}-$ модуль сдвига, коэффициент Пуассона, плотность и скорость сдвиговой волны; $b$ - модуль вектора Бюргерса; $y-$ координата вдоль линии дислокации; $\xi(0, t)=\xi(l, t)=0$; $\mu, \eta$ и $m, q, n, r$ - безразмерные параметры и показатели степени диссипативной и реактивной нелинейности; $m \geq q \geq 0, n \geq r \geq 0 ; \mu|\xi / b|^{m-q}\left|\xi_{t} / C_{\perp}\right|^{q} \ll 1$, $\eta|\xi / b|^{n-r}\left|\xi_{t} / C_{\perp}\right|^{r} \ll 1$.

Функционально структуры введенных в уравнение (1) диссипативного $B \mu|\xi / b|^{m-q}\left|\xi_{t} / C_{\perp}\right|^{q} \xi_{t} \propto \xi_{t}$ и реактивного $C \eta|\xi / b|^{n-r}\left|\xi_{t} / C_{\perp}\right|^{r} \xi_{y y} \propto \xi_{y y}$ нелинейных слагаемых задаются в соответствии с экспериментально наблюдаемыми степенными зависимостями нелинейных коэффициента затухания и фазовой задержки несущей слабой ВЧ волны под действием мощной НЧ волны накачки от ее амплитуды и частоты, при этом параметры нелинейности $\mu, \eta$ и показатели степени $m, q, n, r$ для каждого поликристаллического твердого тела определяются из сравнения аналитических зависимостей НАЭ с результатами эксперимента. Наличие таких слагаемых в уравнении движения дислокации приведет соответственно к диссипативной и реактивной нелинейности уравнения состояния поликристалла, причем последние не будет давать вклада в эффекты АЗВТ, обусловленные НЧ гистерезисной нелинейностью.

Для многих поликристаллических твердых тел (цинк, свинец, гранит, магнезит, мрамор, песчаник, известняк) нелинейные эффекты затухания и фазовой задержки несущей слабой продольной ВЧ волны под действием мощной НЧ продольной волны накачки не зависят от ее частоты, так что для них $q=0, r=0$, однако в общем случае $q \neq 0, r \neq 0$. Довольно разнообразно ведут себя показатели степени $m$ диссипативной нелинейности: для отожженной меди (в зависимости от температуры отжига) $m=1,3 / 2,2$ [20-23], для неотожженного цинка $m=3 / 2$, а для отожженного $-m=2$ [24], для свинца $m=1$ [25], для мрамора $m=2$ [26], для гранита [27] и магнезита $m=1$ [27], для кварцита $m=5 / 4$ [28]. Аналогично ведут себя показатели степени $n$ реактивной нелинейности: для гранита $n=1$ [29], для мрамора $n=3 / 2$ [30], для магнезита $n=1$ [28], для кварцита $n=3 / 2,1 / 2$ [28]. Таким образом, для одного и того же поликристалла, как правило, $m \neq n$ и $q \neq r$. Из различий амплитудно-частотных зависимостей эффектов затухания и фазовой задержки несущей слабой ВЧ волны под действием мощной НЧ волны накачки следует, что механизмы диссипативной и реактивной нелинейностей поликристаллических твердых тел различны. Здесь диссипативная и реактивная нелинейности поликристалла связаны с вынужденным (под действием интенсивной упругой волны) колебательным консервативным движением (скольжением) дислокаций. С одной стороны, такое движение сопровождаются периодическими разрывами „старых“ и образованием „новых“ атомных связей (вблизи линии дислокации), на что затрачивается энергия упругой волны. С другой стороны, вынужденные колебания дислокаций сопровождаются периодическими изменениями их эффективного натяжения. Эти процессы приводят соответственно к диссипативной и к реактивной дислокационной нелинейности поликристалла.

Для получения уравнения состояния поликристалла определим его сдвиговую деформацию $\gamma$ под действием сдвигового напряжения $\tau$ [7-9]:

$$
\gamma(\tau)=(\tau / G)+\gamma_{d i s}(\tau)
$$

где

$$
\gamma_{d i s}(\tau)=b \int_{0}^{\infty} \int_{0}^{l} \xi(y, t) N(l) d y d l
$$

- сдвиговая дислокационная деформация, связанная со смещением $\xi(y, t)$ сегментов дислокаций, $N=N(l)-$ функция распределения сегментов дислокаций по длинам $l, \int_{0}^{\infty} l N(l) d l=\Lambda$ - плотность дислокаций.

Решение уравнения (1) будем искать методом возмущений, полагая при этом, что вынужденные колебания сегмента дислокации на основной моде являются доминирующими:

$$
\xi(y, t) \cong\left[\xi_{0}(t)+\zeta_{1}(t)\right] \sin \frac{\pi y}{l},\left|\xi_{1}(t)\right| \ll\left|\xi_{0}(t)\right| .
$$

Подставляя (3) в (1), получаем уравнения для $\xi_{0}(t)$ и $\xi_{1}(t)$ :

$$
\begin{gathered}
\ddot{\zeta}_{0}+d_{0} \dot{\xi}_{0}+\Omega^{2} \xi_{0}=(4 b / \pi A) \tau \\
\ddot{\zeta}_{1}+d_{0} \dot{\zeta}_{1}+\Omega^{2} \xi_{1}=-\delta d_{0}\left|\xi_{0}\right|^{m-q}\left|\dot{\zeta}_{0}\right|^{q} \dot{\zeta}_{0}+g \Omega^{2}\left|\xi_{0}\right|^{n-r}\left|\dot{\zeta}_{0}\right|^{r} \xi_{0}
\end{gathered}
$$

где

$$
\begin{gathered}
\delta=\frac{2 \mu}{\pi^{1 / 2} b^{m-q} C_{\perp}^{q}} \frac{\Gamma[(m+3) / 2]}{\Gamma[(m+4) / 2]}, \\
g=\frac{2 \eta}{\pi^{1 / 2} b^{n-r} C_{\perp}^{r}} \frac{\Gamma[(n+3) / 2]}{\Gamma[(n+4) / 2]}, \\
\Omega=(\pi / l)(C / A)^{1 / 2}=[2 /(1-v)]^{1 / 2}\left(C_{\perp} / l\right)
\end{gathered}
$$

- резонансная частота основной моды колебания сегмента дислокации длины $l, d_{0}=B / A$ - параметр демпфирования, $\Gamma(m)$ - гамма-функция. (Для меди резонансная частота $\Omega$ сегмента дислокации длиной $l=10^{-5} \mathrm{~m}$ является достаточно высокой и составляет $3.9 \cdot 10^{8} \mathrm{rad} / \mathrm{s}[6,7]$.) 
Решения уравнений (4), (5) имеют вид [32]:

$$
\begin{gathered}
\xi_{0}(t)=\frac{8 b}{\pi \lambda A} \int_{-\infty}^{t} \tau\left(t_{1}\right) \exp \left(\frac{d_{0}}{2}\left(t_{1}-t\right)\right) \sin \left(\frac{\lambda}{2}\left(t-t_{1}\right)\right) d t_{1} \\
\dot{\zeta}_{0}(t)=-\frac{4 b}{\pi \lambda A} \int_{-\infty}^{t} \tau\left(t_{1}\right) \exp \left(\frac{d_{0}}{2}\left(t_{1}-t\right)\right) \\
\times\left[d_{0} \sin \left(\frac{\lambda}{2}\left(t-t_{1}\right)\right)-\lambda \cos \left(\frac{\lambda}{2}\left(t-t_{1}\right)\right)\right] d t_{1},
\end{gathered}
$$

где $\quad D_{\tau}(t)=\left|\xi_{0}(t)\right|^{m-q}\left|\dot{\zeta}_{0}(t)\right|^{q}, \quad G_{\tau}(t)=\left|\xi_{0}(t)\right|^{n-r}\left|\dot{\zeta}_{0}(t)\right|^{r}$, $\lambda^{2}=4 \Omega^{2}-d_{0}^{2}$.

Подставляя (3), (6) в уравнение (2) получаем зависимость $\gamma=\gamma(\tau)$ для сдвиговых напряжений $\tau$ и деформаций $\gamma$ поликристалла:

$$
\begin{aligned}
& \gamma(\tau)=\frac{\tau}{G}+\frac{16}{\pi^{3} \rho} \int_{0}^{\infty} \int_{-\infty}^{t} \tau\left(t_{1}\right) \exp \left(\frac{d_{0}}{2}\left(t_{1}-t\right)\right) \\
& \times \sin \left(\frac{\lambda}{2}\left(t-t_{1}\right)\right) d t_{1} \frac{l N(l) d l}{\lambda}+\frac{16}{\pi^{3} \rho} \\
& \times\left[\delta d_{0} \int_{0}^{\infty} \int_{-\infty}^{t} D_{\tau}\left(t_{1}\right) \tau\left(t_{2}\right) \exp \left(\frac{d_{0}}{2}\left(t_{2}-t\right)\right)\right. \\
& \times\left\{d_{0} \sin \left(\frac{\lambda}{2}\left(t_{1}-t_{2}\right)\right)-\lambda \cos \left(\frac{\lambda}{2}\left(t_{1}-t_{2}\right)\right)\right\} \\
& \times \sin \left(\frac{\lambda}{2}\left(t-t_{1}\right)\right) d t_{1} d t_{2} \frac{l N(l) d l}{\lambda^{2}} \\
& +2 g \int_{0}^{\infty} \int_{-\infty}^{t} \int_{-\infty}^{t_{1}}\left(t_{1}\right) \tau\left(t_{2}\right) \exp \left(\frac{d_{0}}{2}\left(t_{2}-t\right)\right) \\
& \left.\times \sin \left(\frac{\lambda}{2}\left(t_{1}-t_{2}\right)\right) \sin \left(\frac{\lambda}{2}\left(t-t_{1}\right)\right) d t_{1} d t_{2} \frac{\Omega^{2} l N(l) d l}{\lambda^{2}}\right]
\end{aligned}
$$

где

$$
\begin{aligned}
\left(\begin{array}{l}
D_{\tau}(t) \\
G_{\tau}(t)
\end{array}\right)= & \left(\frac{4}{\pi^{2} b \rho}\right)^{\left(\begin{array}{c}
m \\
n
\end{array}\right) \mid} \mid \frac{2}{\lambda} \int_{-\infty}^{t} \tau\left(t_{1}\right) \exp \left(\frac{d_{0}}{2}\left(t_{1}-t\right)\right) \\
& \times \sin \left(\frac{\lambda}{2}\left(t-t_{1}\right)\right) d t_{1}||^{\left(\begin{array}{c}
m-q \\
n-r
\end{array}\right)} \mid \frac{1}{\lambda} \int_{-\infty}^{t} \tau\left(t_{1}\right) \\
& \times \exp \left(\frac{d_{0}}{2}\left(t_{1}-t\right)\right)\left[d_{0} \sin \left(\frac{\lambda}{2}\left(t-t_{1}\right)\right)\right. \\
& \left.-\lambda \cos \left(\frac{\lambda}{2}\left(t-t_{1}\right)\right)\right]\left.d t_{1}\right|^{\left(\begin{array}{l}
q \\
r
\end{array}\right)} .
\end{aligned}
$$

Переходя в (7) от сдвиговых напряжений $\tau$ и деформаций $\gamma_{d i s}$ к продольным напряжениям $\sigma=\tau / R$ и деформациям $\varepsilon_{d i s}=R \gamma_{d i s}[1,7,8]$, получим аналогичную а зависимость $\varepsilon=\varepsilon(\sigma)$ для продольных напряжений $\sigma$ и деформаций $\varepsilon$ для стержня (вдоль его оси $x$ ):

$$
\begin{aligned}
& \varepsilon(\sigma)=\frac{\sigma}{E}+\frac{16 R^{2}}{\pi^{3} \rho} \int_{0}^{\infty} \int_{-\infty}^{t} \sigma\left(t_{1}\right) \exp \left(\frac{d_{0}}{2}\left(t_{1}-t\right)\right) \\
& \times \sin \left(\frac{\lambda}{2}\left(t-t_{1}\right)\right) d t_{1} \frac{l N(l) d l}{\lambda}+\frac{16 R^{2}}{\pi^{3} \rho} \\
& \times\left[\delta d_{0} \int_{0}^{\infty} \int_{-\infty}^{t} D_{-\infty}^{t_{1}} D_{\sigma}\left(t_{1}\right) \sigma\left(t_{2}\right) \exp \left(\frac{d_{0}}{2}\left(t_{2}-t\right)\right)\right. \\
& \times\left\{d_{0} \sin \left(\frac{\lambda}{2}\left(t_{1}-t_{2}\right)\right)-\lambda \cos \left(\frac{\lambda}{2}\left(t_{1}-t_{2}\right)\right)\right\} \\
& \times \sin \left(\frac{\lambda}{2}\left(t-t_{1}\right)\right) d t_{1} d t_{2} \frac{l N(l) d l}{\lambda^{2}} \\
& +2 g \int_{0}^{\infty} \int_{-\infty}^{t} G_{\sigma}\left(t_{1}\right) \sigma\left(t_{2}\right) \exp \left(\frac{d_{0}}{2}\left(t_{2}-t\right)\right) \\
& \left.\times \sin \left(\frac{\lambda}{2}\left(t_{1}-t_{2}\right)\right) \sin \left(\frac{\lambda}{2}\left(t-t_{1}\right)\right) d t_{1} d t_{2} \frac{\Omega^{2} l N(l) d l}{\lambda^{2}}\right],
\end{aligned}
$$

где

$$
\begin{aligned}
& \left(\begin{array}{l}
D_{\sigma}(t) \\
G_{\sigma}(t)
\end{array}\right)=\left(\frac{4 R}{\pi^{2} b \rho}\right)^{\left(\begin{array}{c}
m \\
n
\end{array}\right)} \mid \frac{2}{\lambda} \int_{-\infty}^{t} \sigma\left(t_{1}\right) \exp \left(\frac{d_{0}}{2}\left(t_{1}-t\right)\right) \\
& \times\left.\sin \left(\frac{\lambda}{2}\left(t-t_{1}\right)\right) d t_{1}\right|^{\left(\begin{array}{c}
m-q \\
n-r
\end{array}\right)} \mid \frac{1}{\lambda} \int_{-\infty}^{t} \sigma\left(t_{1}\right) \\
& \times \exp \left(\frac{d_{0}}{2}\left(t_{1}-t\right)\right)\left[d_{0} \sin \left(\frac{\lambda}{2}\left(t-t_{1}\right)\right)\right. \\
& \left.-\lambda \cos \left(\frac{\lambda}{2}\left(t-t_{1}\right)\right)\right]\left.d t_{1}\right|^{\left(\begin{array}{l}
q \\
r
\end{array}\right)},
\end{aligned}
$$

$E=2 G(1+v)$ - модуль Юнга, $R=R(\theta, \varphi)=\sin \theta \cos \theta$ $\times \cos \varphi$ множитель, учитывающий ориентацию направления распространения продольной волны по отношению к плоскостям и направлениям скольжения дислокации в поликристалле, $\theta-$ угол между осью стержня и нормалью к плоскости скольжения дислокации, $\varphi$ - угол между направлением скольжения дислокации и проекцией оси стержня на плоскость скольжения, $0 \leq \theta \leq \pi / 2,|\varphi| \leq \pi / 2$ [1]. (Для изо- 
тропных поликристаллов $\langle R(\theta, \varphi)\rangle=\frac{4}{3 \pi^{2}} \approx 1.35 \cdot 10^{-1}$, $\left.\left\langle R^{2}(\theta, \varphi)\right\rangle=\frac{4}{15 \pi} \approx 8.5 \cdot 10^{-2}\right)$.

При малой Плотности дислокаций, когда выполняется условие

$$
\begin{aligned}
& \frac{16 R^{2} C_{0}^{2}}{\pi^{3}} \mid \int_{0}^{\infty} \int_{-\infty}^{t} \varepsilon\left(t_{1}\right) \exp \left(\frac{d_{0}}{2}\left(t_{1}-t\right)\right) \\
& \times \sin \left(\frac{\lambda}{2}\left(t-t_{1}\right)\right) d t_{1} \frac{l N(l) d l}{\lambda}|\ll| \varepsilon \mid \ll 1,
\end{aligned}
$$

из (8) получаем уравнение состояния $\sigma=\sigma(\varepsilon)$ для стержня в „каноническом“ виде

$$
\begin{aligned}
& \sigma(\varepsilon)=E\left[\varepsilon-\frac{16 R^{2} C_{0}^{2}}{\pi^{3}} \int_{0}^{\infty} \int_{-\infty}^{t} \varepsilon\left(t_{1}\right) \exp \left(\frac{d_{0}}{2}\left(t_{1}-t\right)\right)\right. \\
& \left.\times \sin \left(\frac{\lambda}{2}\left(t-t_{1}\right)\right) d t_{1} \frac{l N(l) d l}{\lambda}\right]-E \frac{16 R^{2} C_{0}^{2}}{\pi^{3}} \\
& \times\left[\delta d_{0} \int_{0}^{\infty} \int_{-\infty}^{t} D_{\varepsilon}\left(t_{1}\right) \varepsilon\left(t_{2}\right) \exp \left(\frac{d_{0}}{2}\left(t_{2}-t\right)\right)\right. \\
& \times\left\{d_{0} \sin \left(\frac{\lambda}{2}\left(t_{1}-t_{2}\right)\right)-\lambda \cos \left(\frac{\lambda}{2}\left(t_{1}-t_{2}\right)\right)\right\} \\
& \times \sin \left(\frac{\lambda}{2}\left(t-t_{1}\right)\right) d t_{1} d t_{2} \frac{l N(l) d l}{\lambda^{2}} \\
& \left.\times \sin \left(\frac{\lambda}{2}\left(t_{1}-t_{2}\right)\right) \sin \left(\frac{\lambda}{2}\left(t-t_{1}\right)\right) d t_{1} d t_{2} \frac{\Omega^{2} l N(l) d l}{\lambda^{2}}\right] \\
& +2 g\left(\int_{0}^{\infty} \int_{-\infty}^{t_{1}} G_{\varepsilon}\left(t_{1}\right) \varepsilon\left(t_{2}\right) \exp \left(\frac{d_{0}}{2}\left(t_{2}-t\right)\right)\right.
\end{aligned}
$$

где $C_{0}=(E / \rho)^{1 / 2}$,

$$
\begin{aligned}
& \left(\begin{array}{l}
D_{\varepsilon}(t) \\
G_{\varepsilon}(t)
\end{array}\right)=\left(\frac{4 R C_{0}^{2}}{\pi^{2} b}\right)^{\left(\begin{array}{c}
m \\
n
\end{array}\right) \mid} \mid \frac{2}{\lambda} \int_{-\infty}^{t} \varepsilon\left(t_{1}\right) \exp \left(\frac{d_{0}}{2}\left(t_{1}-t\right)\right) \\
& \times\left.\sin \left(\frac{\lambda}{2}\left(t-t_{1}\right)\right) d t_{1}\right|^{\left(\begin{array}{c}
m-q \\
n-r
\end{array}\right)} \mid \frac{1}{\lambda} \int_{-\infty}^{t} \varepsilon\left(t_{1}\right) \\
& \times \exp \left(\frac{d_{0}}{2}\left(t_{1}-t\right)\right)\left[d_{0} \sin \left(\frac{\lambda}{2}\left(t-t_{1}\right)\right)\right. \\
& \left.-\lambda \cos \left(\frac{\lambda}{2}\left(t-t_{1}\right)\right)\right]\left.d t_{1}\right|^{\left(\begin{array}{l}
q \\
r
\end{array}\right)} .
\end{aligned}
$$

Заметим, что в отличие от традиционно используемых выражений для линейных декремента затухания и дефекта модуля упругости [7-12], нелинейное уравнение состояния (9) поликристалла наиболее полно определяет его акустические свойства, поскольку именно уравнение состояния позволяет в полной мере исследовать нелинейные волновые процессы в поликристалле с дислокациями и определить не только линейные, но и нелинейные выражения для декремента затухания и дефекта модуля упругости, а также и любые другие характеристики нелинейного взаимодействия упругих волн.

В НЧ приближении, когда $\omega \ll \Omega, \omega d_{0} \ll \Omega^{2}(\omega-$ частота волны), уравнение состояния (6) имеет простой вид, при этом, поскольку $\left(d_{0} / \Omega^{2}\right)|\dot{\varepsilon}| \ll|\varepsilon|$, диссипативная и реактивная нелинейности входят практически раздельно:

$$
\begin{aligned}
& \sigma(\varepsilon)=E\left\{\left(1-\frac{8 R^{2} C_{0}^{2}}{\pi^{3}} \int_{0}^{\infty} \frac{l N(l) d l}{\Omega^{2}}\right) \varepsilon+\frac{8 R^{2} C_{0}^{2}}{\pi^{3}}\right. \\
& \left.\times\left(\int_{0}^{\infty} \frac{l N(l) d l}{\Omega^{2}}\right) d_{0} \dot{\varepsilon}\right\}+E \frac{8 R^{2} C_{0}^{2}}{\pi^{3}}\left[\left(\frac{4 R C_{0}^{2}}{\pi^{2} b}\right)^{m}\right. \\
& \times \delta d_{0}|\dot{\varepsilon}|^{q} \dot{\varepsilon} \int_{0}^{\infty}\left|\varepsilon-\frac{d_{0}}{\Omega^{2}} \dot{\varepsilon}\right|^{m-q} \frac{l N(l) d l}{\Omega^{2(m+2)}}-\left(\frac{4 R C_{0}^{2}}{\pi^{2} b}\right)^{n} \\
& \left.\quad \times g|\dot{\varepsilon}|^{r} \int_{0}^{\infty}\left(\varepsilon-\frac{2 d_{0}}{\Omega^{2}} \dot{\varepsilon}\right)\left|\varepsilon-\frac{d_{0}}{\Omega^{2}} \dot{\varepsilon}\right|^{n-r} \frac{l N(l) d l}{\Omega^{2(n+2)}}\right] .
\end{aligned}
$$

Из этого уравнения видно, что структура диссипативной и реактивной нелинейностей поликристалла повторяет структуру соответствующих нелинейных слагаемых в уравнении (1) движения дислокации. В НЧ диапазоне (при $\delta, g>0$ ) при увеличении амплитуды волны коэффициент поглощения волны увеличивается, а ее фазовая скорость - уменьшается. В общем же случае каждая нелинейность (и диссипативная, и реактивная) приводит как к нелинейному поглощению волны, так и к нелинейному изменению ее фазовой скорости. Из анализа амплитудно-частотных зависимостей НАЭ и сравнения их с результатами соответствующих экспериментов можно определить параметры дислокационной нелинейности и эффективные характеристики дислокационной структуры поликристаллических твердых тел.

Подставляя уравнение состояния (9) в уравнение движения $\rho U_{t t}=\sigma_{x}(\varepsilon)$ [31], где $U=U(x, t)$ - продольное (вдоль оси $x$ ) смещение, $\varepsilon=\partial U(x, t) / \partial x$, получим нелинейное волновое уравнение для деформации $\varepsilon(x, t)$, определяющее волновые процессы в стержне с дислока- 
ционной диссипативной и реактивной нелинейностью:

$$
\begin{aligned}
& \varepsilon_{t t}(x, t)-C_{0}^{2} \varepsilon_{x x}(x, t)=-\frac{16 R^{2} C_{0}^{4}}{\pi^{3}} \int_{0}^{\infty} \int_{-\infty}^{t} \varepsilon_{x x}\left(x, t_{1}\right) \\
& \times \exp \left(\frac{d_{0}}{2}\left(t_{1}-t\right)\right) \sin \left(\frac{\lambda}{2}\left(t-t_{1}\right)\right) d t_{1} \frac{l N(l) d l}{\lambda} \\
& -\frac{16 R^{2} C_{0}^{4}}{\pi^{3}} \delta d_{0} \int_{0}^{\infty} \int_{-\infty}^{t} \int_{-\infty}^{t_{1}} D_{\varepsilon}\left(x, t_{1}\right) \varepsilon\left(x, t_{2}\right) \\
& \times \exp \left(\frac{d_{0}}{2}\left(t_{2}-t\right)\right)\left\{d_{0} \sin \left(\frac{\lambda}{2}\left(t_{1}-t_{2}\right)\right)\right. \\
& \left.-\lambda \cos \left(\frac{\lambda}{2}\left(t_{1}-t_{2}\right)\right)\right\} \sin \left(\frac{\lambda}{2}\left(t-t_{1}\right)\right) d t_{1} d t_{2} \frac{l N(l) d l}{\lambda^{2}} \\
& +2 g \int_{0}^{\infty} \int_{-\infty}^{t} \int_{-\infty}^{t_{1}} G_{\varepsilon}\left(x, t_{1}\right) \varepsilon\left(x, t_{2}\right) \exp \left(\frac{d_{0}}{2}\left(t_{2}-t\right)\right) \\
& \left.\times \sin \left(\frac{\lambda}{2}\left(t_{1}-t_{2}\right)\right) \sin \left(\frac{\lambda}{2}\left(t-t_{1}\right)\right) d t_{1} d t_{2} \frac{\Omega^{2} l N(l) d l}{\lambda^{2}}\right]_{x x} .
\end{aligned}
$$

В линейном приближении из уравнения (11) следуют дисперсионное соотношение $\kappa=\kappa(\omega)$, выражения для фазовой скорости $C(\omega)=\omega / \kappa(\omega)$ и коэффициента затухания $\alpha=\alpha(\omega)$ слабой гармонической волны $\varepsilon(x, t) \propto \exp \{j[\omega t-\kappa(\omega) x]-\alpha(\omega) x\}$, соответствующие результатам линейной части дислокационной теории поглощения Гранато-Люкке [7-9]:

$$
\begin{gathered}
\kappa(\omega)=\frac{\omega}{C_{0}}\left(1+\frac{4 R^{2} C_{0}^{2}}{\pi^{3}} \int_{0}^{\infty} \frac{\left(\Omega^{2}-\omega^{2}\right) l N(l) d l}{\left(\Omega^{2}-\omega^{2}\right)^{2}+d_{0}^{2} \omega^{2}}\right), \\
C(\omega)=\frac{C_{0}}{1+\frac{4 R^{2} C_{0}^{2}}{\pi^{3}} \int_{0}^{\infty} \frac{\left(\Omega^{2}-\omega^{2}\right) l N(l) d l}{\left(\Omega^{2}-\omega^{2}\right)^{2}+d_{0}^{2} \omega^{2}}} \\
\approx C_{0}\left(1-\frac{4 R^{2} C_{0}^{2}}{\pi^{3}} \int_{0}^{\infty} \frac{\left(\Omega^{2}-\omega^{2}\right) l N(l) d l}{\left(\Omega^{2}-\omega^{2}\right)^{2}+d_{0}^{2} \omega^{2}}\right), \\
\alpha(\omega)=\frac{4 R^{2} C_{0}}{\pi^{3}} d_{0} \omega^{2} \int_{0}^{\infty} \frac{l N(l) d l}{\left(\Omega^{2}-\omega^{2}\right)^{2}+d_{0}^{2} \omega^{2}},
\end{gathered}
$$

где $\kappa(\omega)-$ волновое число для волны с частотой $\omega$.

\section{2. Влияние статического напряжения на затухание и фазовую задержку несущей упругой гармонической волны}

Вначале рассмотрим эффекты, связанные с влиянием продольного (вдоль стержня) статического напряжения $\sigma_{x x}=\sigma_{0}=$ const (при котором еще не возникает пластической деформации поликристалла и размножения в нем дислокаций [1-6]) на затухание и фазовую задержку несущей слабой (пробной) продольной гармонической волны в стержне. Такие эффекты, линейные по амплитуде слабой волны, имеют место при $q=r=0$, при этом слабая волна распространяется линейно: скорость и затухание волны не зависят от ее амплитуды.

Подставляя в уравнение $(11) \varepsilon(x, t)=\varepsilon_{0}+\varepsilon_{1}(x, t)$, $\varepsilon_{0}=\sigma_{0} / E-$ продольная статическая упругая деформация, $\varepsilon_{1}(x, t)=a(x) \cos [\omega t-k x-\varphi(x)], \varepsilon_{1}(x=0, t)=$ $=a_{0} \cos \omega t, \quad k=\omega / C_{0}, \quad\left|\varepsilon_{1}(x, t)\right| \ll\left|\varepsilon_{0}\right|, \quad d a(x) / d x \ll k a(x)$, $d \varphi(x) / d x \ll k \varphi(x), \quad \frac{a(x)}{\left|\varepsilon_{0}\right|} \ll \frac{1}{\sqrt{1+\left(\omega / d_{0}\right)^{2}}}$, и выделяя в нем слагаемые на частоте $\omega$, получим уравнения для амплитуды $a(x)$ и фазы $\varphi(x)$ волны $\varepsilon_{1}(x, t)$ :

$$
\begin{aligned}
& \frac{1}{a(x)} \frac{d a(x)}{d x}=-\frac{4 R^{2} C_{0}}{\pi^{3}} Z(\omega)-\mu P_{0}\left|\varepsilon_{0}\right|^{m} d_{0} \omega^{2} \\
& \times \int_{0}^{\infty} \frac{\left[\left(\Omega^{2}-\omega^{2}\right)^{2}-d_{0}^{2} \omega^{2}\right] l N(l) d l}{\Omega^{2 m}\left[\left(\Omega^{2}-\omega^{2}\right)^{2}+d_{0}^{2} \omega^{2}\right]^{2}}-2 \eta Q_{0}\left|\varepsilon_{0}\right|^{n} d_{0} \omega^{2} \\
& \times \int_{0}^{\infty} \frac{\left(\Omega^{2}-\omega^{2}\right) l N(l) d l}{\Omega^{2(n-1)}\left[\left(\Omega^{2}-\omega^{2}\right)^{2}+d_{0}^{2} \omega^{2}\right]^{2}} \\
& \frac{d \varphi(x)}{d x}=\frac{4 R^{2} C_{0}}{\pi^{3}} W(\omega)-2 \mu P_{0}\left|\varepsilon_{0}\right|^{m} d_{0}^{2} \omega^{3} \\
& \quad \times \int_{0}^{\infty} \frac{\left(\Omega^{2}-\omega^{2}\right) l N(l) d l}{\Omega^{2 m}\left[\left(\Omega^{2}-\omega^{2}\right)^{2}+d_{0}^{2} \omega^{2}\right]^{2}}+\eta Q_{0}\left|\varepsilon_{0}\right|^{n} \omega \\
& \quad \times \int_{0}^{\infty} \frac{\left[\left(\Omega^{2}-\omega^{2}\right)^{2}-d_{0}^{2} \omega^{2}\right] l N(l) d l}{\Omega^{2(n-1)}\left[\left(\Omega^{2}-\omega^{2}\right)^{2}+d_{0}^{2} \omega^{2}\right]^{2}}
\end{aligned}
$$

где

$$
\begin{gathered}
Z(\omega)=d_{0} \omega^{2} \int_{0}^{\infty} \frac{l N(l) d l}{\left(\Omega^{2}-\omega^{2}\right)^{2}+d_{0}^{2} \omega^{2}}, \\
W(\omega)=\omega \int_{0}^{\infty} \frac{\left(\Omega^{2}-\omega^{2}\right) l N(l) d l}{\left(\Omega^{2}-\omega^{2}\right)^{2}+d_{0}^{2} \omega^{2}}, \\
P_{0}=\frac{8 R^{2} C_{0}}{\pi^{7 / 2}}\left(\frac{4 R C_{0}^{2}}{\pi^{2} b^{2}}\right)^{m} \frac{\Gamma[(m+3) / 2]}{\Gamma[(m+4) / 2]}, \\
Q_{0}=\frac{8 R^{2} C_{0}}{\pi^{7 / 2}}\left(\frac{4 R C_{0}^{2}}{\pi^{2} b^{2}}\right)^{n} \frac{\Gamma[(n+3) / 2](n+1)}{\Gamma[(m+4) / 2]} .
\end{gathered}
$$

Из уравнений (13), (14) находим выражения для коэффициента затухания $\xi\left(\varepsilon_{0}, \omega\right)=\ln \left[a(0, x=L) / a\left(\varepsilon_{0}\right.\right.$, $x=L)] \quad$ и фазовой задержки несущей $\tau\left(\varepsilon_{0}, \omega\right)=$ $=\left[\varphi\left(\varepsilon_{0}, x=L\right)-\varphi(0, x=L)\right] / \omega \quad$ слабой $\quad$ волны $\quad$ при $x=L$, обусловленные диссипативной и реактивной 
нелинейностью поликристалла:

$$
\begin{gathered}
\chi\left(\varepsilon_{0}, \omega\right)=\mu P_{0}\left|\varepsilon_{0}\right|^{m} L d_{0} \omega^{2} \int_{0}^{\infty} \frac{\left[\left(\Omega^{2}-\omega^{2}\right)^{2}-d_{0}^{2} \omega^{2}\right] l N(l) d l}{\Omega^{2 m}\left[\left(\Omega^{2}-\omega^{2}\right)^{2}+d_{0}^{2} \omega^{2}\right]^{2}} \\
+2 \eta Q_{0}\left|\varepsilon_{0}\right|^{n} L d_{0} \omega^{2} \int_{0}^{\infty} \frac{\left(\Omega^{2}-\omega^{2}\right) l N(l) d l}{\Omega^{2(n-1)}\left[\left(\Omega^{2}-\omega^{2}\right)^{2}+d_{0}^{2} \omega^{2}\right]^{2}}, \quad(15) \\
\tau\left(\varepsilon_{0}, \omega\right)=-2 \mu P_{0}\left|\varepsilon_{0}\right|^{m} L d_{0}^{2} \omega^{2} \int_{0}^{\infty} \frac{\left(\Omega^{2}-\omega^{2}\right) l N(l) d l}{\Omega^{2 m}\left[\left(\Omega^{2}-\omega^{2}\right)^{2}+d_{0}^{2} \omega^{2}\right]^{2}} \\
+\eta Q_{0}\left|\varepsilon_{0}\right|^{n} L \int_{0}^{\infty} \frac{\left[\left(\Omega^{2}-\omega^{2}\right)^{2}-d_{0}^{2} \omega^{2}\right] l N(l) d l}{\Omega^{2(n-1)}\left[\left(\Omega^{2}-\omega^{2}\right)^{2}+d_{0}^{2} \omega^{2}\right]^{2}} .
\end{gathered}
$$

Из уравнений (13)-(16) видно, что диссипативная и реактивная нелинейности приводят к изменениям амплитуды и фазы несущей пробной волны под действием статической нагрузки, при этом эффективные параметры нелинейности поликристалла зависят от частоты волны. Таким образом, по измеренным в эксперименте зависимостям $\chi\left(\varepsilon_{0}, \omega\right)$ и $\tau\left(\varepsilon_{0}, \omega\right)$ от $\varepsilon_{0}$ и $\omega$ можно определить параметры уравнения движения дислокации $\left(\mu, \eta, m, n, d_{0}\right)$ и функцию распределения $N=N(l)$ дислокаций.

Приведем оценки для параметров $\mu$ и $\eta$ диссипативной и реактивной нелинейностей дислокации для изотропного поликристалла $\left(\langle R(\theta, \varphi)\rangle=\frac{4}{3 \pi^{2}} \approx 1.35 \cdot 10^{-1}\right.$, $\left.\left\langle R^{2}(\theta, \varphi)\right\rangle=\frac{4}{15 \pi} \approx 8.5 \cdot 10^{-2}\right)$ при $m=n=1, q=r=0$, $b=3 \cdot 10^{-8} \mathrm{~cm}, \quad N(l)=\left(\Lambda / l_{0}\right) \delta\left(l-l_{0}\right), \quad \Lambda=10^{11} \mathrm{~m}^{-2}$, $l_{0}=10^{-5} \mathrm{~m}, \quad C_{\perp}=2.3 \cdot 10^{3} \mathrm{~m} / \mathrm{s}, \quad C_{0}=3.7 \cdot 10^{3} \mathrm{~m} / \mathrm{s}$, $\Omega \approx 3.9 \cdot 10^{8} \mathrm{rad} / \mathrm{s}, \quad v=0.3, \quad d_{0}=10^{8} \mathrm{rad} / \mathrm{s}, \quad \varepsilon_{0}=10^{-5}$, $x=L=0.3 \mathrm{~m}, \quad T=L / C_{0} \approx 8 \cdot 10^{-5} \mathrm{~s}, \quad \chi\left(\varepsilon_{0}, \omega\right)=1$, $\tau\left(\varepsilon_{0}, \omega\right) / T=10^{-2}$. Полагая, что при распространении в таком поликристалле пробной волны с частотой $\omega=2 \pi f \ll \Omega, f=500 \mathrm{kHz}$ ее нелинейное затухание возникает только за счет диссипативной нелинейности $(\eta=0)$, а фазовая задержка несущей - только за счет реактивной нелинейности $(\mu=0)$, получим: $\mu \approx 2 \cdot 10^{-1}$ и $\eta \approx 3.7 \cdot 10^{-4}$.

\section{3. Затухание и фазовая задержка несущей слабой высокочастотной волны под действием сильной низкочастотной волны в резонаторе}

Исследуем нелинейные эффекты, связанные с влиянием сильной резонансной стоячей НЧ волны накачки на затухание и скорость распространение слабого ВЧ импульса в стержне, когда для НЧ волны накачки стержень является резонатором, а для ВЧ импульса - безграничной средой. (Эффекты такого влияния наблюдались в стержнях из многих поликристаллических металлов и горных пород [20-30].
При возбуждении сильной резонансной НЧ волны накачки в резонаторе на частоте $F \approx 3 \mathrm{kHz}$ с амплитудой деформации $\varepsilon_{m} \approx 10^{-5}$ амплитуда ВЧ импульса с несущей частотой $f=\omega / 2 \pi \approx 300 \mathrm{kHz}$ уменьшалась в несколько раз, а относительная фазовая задержка его несущей достигала $1 \%$.) Для резонатора с жесткой $(x=0)$ и мягкой $(x=L)$ границами выражение для деформации НЧ волны накачки (в резонансе) имеет вид: $\varepsilon_{1}(x, t)=\varepsilon_{m} \cos K_{p} x \cos \left(\Omega_{p} t+\theta\right)$, где $K_{p} L=\pi(2 p-1) / 2, L-$ длина стержня, $\Omega_{p}=C_{0} K_{p}$, $p$ - номер продольной моды резонатора, $\theta=$ const.

Решение волнового уравнения (11) будем искать в виде $\varepsilon(x, t)=\varepsilon_{1}(x, t)+\varepsilon_{2}(x, t), \quad \varepsilon_{2}(x, t)=$ $=a(x) \cos [\omega t-k x-\varphi(x)], \quad \varepsilon_{m} \gg a(x), \quad \varepsilon_{2}(x=0, t)=$ $=a_{0} \cos \omega t, \Omega_{p} \varepsilon_{m} \gg \omega a(x), \omega \gg \Omega_{p}, k=\omega / C_{0} \gg K_{p}$, $d a(x) / d x \ll k a(x), d \varphi(x) / d x \ll k \varphi(x)$. Здесь, однако, выражения для интегралов $I_{1,2}\left[\varepsilon\left(x, t_{1}\right)\right]$ в степенных модульных (вообще говоря, неаналитических) множителях $D_{\varepsilon}\left(x t_{1}\right)$ и $G_{\varepsilon}\left(x, t_{1}\right)$ в уравнении необходимо преобразовать к „мультипликативному“ (квазигармоническому) виду [33]:

$$
\begin{aligned}
& I_{1}\left[\varepsilon\left(x, t_{1}\right)\right]=\frac{2}{\lambda} \int_{-\infty}^{t_{1}} \varepsilon\left(t_{2}\right) \exp \left(\frac{d_{0}}{2}\left(t_{2}-t_{1}\right)\right) \sin \left(\frac{\lambda}{2}\left(t_{1}-t_{2}\right)\right) \\
& \times d t_{2}=\frac{\varepsilon_{m} \cos K_{p} x \cos \left(\Omega_{p} t_{1}+\theta-\Psi_{1}\right)}{\left[\left(\Omega^{2}-\Omega_{p}^{2}\right)^{2}+d_{0}^{2} \Omega_{p}^{2}\right]^{1 / 2}} \\
& +\frac{a(x) \cos \left[\omega t_{1}-k x-\varphi(x)-\Psi_{2}\right]}{\left[\left(\Omega^{2}-\omega^{2}\right)^{2}+d_{0}^{2} \omega^{2}\right]^{1 / 2}} \\
& =\quad \in_{m}\left(x, t_{1}\right) \cos \left[\Omega_{p} t_{1}+\theta-\Psi_{1}+\Phi_{1}\left(x, t_{1}\right)\right], \\
& I_{2}\left[\varepsilon\left(x, t_{1}\right)\right]=\frac{1}{\lambda} \int_{-\infty}^{t_{1}} \varepsilon\left(t_{2}\right) \exp \left(\frac{d_{0}}{2}\left(t_{2}-t_{1}\right)\right) \\
& \quad \times\left[d_{0} \sin \left(\frac{\lambda}{2}\left(t_{1}-t_{2}\right)\right)-\lambda \cos \left(\frac{\lambda}{2}\left(t_{1}-t_{2}\right)\right)\right] d t_{2} \\
& =-\frac{\varepsilon_{m} \Omega_{p} \cos K_{p} x \sin \left(\Omega_{p} t_{1}+\theta-\Psi_{1}\right)}{\left[\left(\Omega^{2}-\Omega_{p}^{2}\right)^{2}+d_{0}^{2} \Omega_{p}^{2}\right]^{1 / 2}} \\
& \quad-\frac{a(x) \omega \sin \left[\omega t_{1}-k x-\varphi(x)-\Psi_{2}\right]}{\left[\left(\Omega^{2}-\omega^{2}\right)^{2}+d_{0}^{2} \omega^{2}\right]^{1 / 2}} \\
& \left.\quad t_{1}\right) \sin \left[\Omega_{p} t_{1}+\theta-\Psi_{1}+\Phi_{2}\left(x, t_{1}\right)\right],
\end{aligned}
$$

где

$$
\begin{aligned}
& \in_{m}\left(x, t_{1}\right) \approx \frac{\varepsilon_{m}\left|\cos K_{p} x\right|}{\left[\left(\Omega^{2}-\Omega_{p}^{2}\right)^{2}+d_{0}^{2} \Omega_{p}^{2}\right]^{1 / 2}} \\
& \times\left(1+\frac{a(x) \cos \left[\Delta \Psi\left(x, t_{1}\right)\right]}{\varepsilon_{m} \cos K_{p} x} \sqrt{\frac{\left(\Omega^{2}-\Omega_{p}^{2}\right)^{2}+d_{0}^{2} \Omega_{p}^{2}}{\left(\Omega^{2}-\omega^{2}\right)^{2}+d_{0}^{2} \omega^{2}}}\right), \\
& \Delta \Psi\left(x, t_{1}\right)=\left[\omega t_{1}-k x-\varphi(x)-\Psi_{2}\right]-\left[\Omega_{p} t_{1}+\theta-\Psi_{1}\right],
\end{aligned}
$$




$$
\begin{gathered}
\Psi_{1}=\operatorname{arctg}\left(\frac{d_{0} \Omega_{p}}{\Omega^{2}-\Omega_{p}^{2}}\right), \quad \Psi_{2}=\operatorname{arctg}\left(\frac{d_{0} \omega}{\Omega^{2}-\omega^{2}}\right), \\
\Phi_{1}\left(x, t_{1}\right) \approx \frac{a(x) \sin \left[\Delta \Psi\left(x, t_{1}\right)\right]}{\varepsilon_{m} \cos K_{p} x} \sqrt{\frac{\left(\Omega^{2}-\Omega_{p}^{2}\right)^{2}+d_{0}^{2} \Omega_{p}^{2}}{\left(\Omega^{2}-\omega^{2}\right)^{2}+d_{0}^{2} \omega^{2}}} \ll 1, \\
S_{m}\left(x, t_{1}\right) \approx \frac{\varepsilon_{m} \Omega_{p}\left|\cos K_{p} x\right|}{\left[\left(\Omega^{2}-\Omega_{p}^{2}\right)^{2}+d_{0}^{2} \Omega_{p}^{2}\right]^{1 / 2}} \\
\times\left(1+\frac{a(x) \omega \cos \left[\Delta \Psi\left(x, t_{1}\right)\right]}{\varepsilon_{m} \Omega_{p} \cos K_{p} x} \sqrt{\frac{\left(\Omega^{2}-\Omega_{p}^{2}\right)^{2}+d_{0}^{2} \Omega_{p}^{2}}{\left(\Omega^{2}-\omega^{2}\right)^{2}+d_{0}^{2} \omega^{2}}}\right), \\
\Phi_{2}\left(x, t_{1}\right) \approx \frac{a(x) \omega \sin \left[\Delta \Psi\left(x, t_{1}\right)\right]}{\varepsilon_{m} \Omega_{p} \cos K_{p} x} \sqrt{\frac{\left(\Omega^{2}-\Omega_{p}^{2}\right)^{2}+d_{0}^{2} \Omega_{p}^{2}}{\left(\Omega^{2}-\omega^{2}\right)^{2}+d_{0}^{2} \omega^{2}}} \ll 1, \\
\frac{a(x) \omega}{\varepsilon_{m} \Omega_{p}\left|\cos K_{p} x\right|} \sqrt{\frac{\left(\Omega^{2}-\Omega_{p}^{2}\right)^{2}+d_{0}^{2} \Omega_{p}^{2}}{\left(\Omega^{2}-\omega^{2}\right)^{2}+d_{0}^{2} \omega^{2}}} \ll 1, \\
\left|\Phi_{2}\left(x, t_{1}\right)-\Phi_{1}\left(x, t_{1}\right)\right| \ll 1 .
\end{gathered}
$$

Подставляя выражения (17), (18) в уравнение (11) и выделяя в нем слагаемые на частоте $\omega$, получим уравнения для амплитуды $a(x)$ и фазы $\varphi(x)$ слабой ВЧ волны:

$$
\begin{aligned}
& \frac{1}{a(x)} \frac{d a(x)}{d x}=-\frac{4 R^{2} C_{0}}{\pi^{3}} d_{0} \omega^{2} \int_{0}^{\infty} \frac{l N(l) d l}{\left[\left(\Omega^{2}-\omega^{2}\right)^{2}+d_{0}^{2} \omega^{2}\right]} \\
& -\mu P \varepsilon_{m}^{m}\left|\cos K_{p} x\right|^{m} \Omega_{p}^{q} d_{0} \omega^{2} \\
& \quad \times \int_{0}^{\infty} \frac{\left[\left(\Omega^{2}-\omega^{2}\right)^{2}-d_{0}^{2} \omega^{2}\right] l N(l) d l}{\left[\left(\Omega^{2}-\Omega_{p}^{2}\right)^{2}+d_{0}^{2} \Omega_{p}^{2}\right]^{m / 2}\left[\left(\Omega^{2}-\omega^{2}\right)^{2}+d_{0}^{2} \omega^{2}\right]^{2}} \\
& -2 \eta Q \varepsilon_{m}^{n}\left|\cos K_{p} x\right|^{n} \Omega_{p}^{r} d_{0} \omega^{2} \\
& \quad \times \int_{0}^{\infty} \frac{\left(\Omega^{2}-\omega^{2}\right) \Omega^{2} l N(l) d l}{\left[\left(\Omega^{2}-\Omega_{p}^{2}\right)^{2}+d_{0}^{2} \Omega_{p}^{2}\right]^{n / 2}\left[\left(\Omega^{2}-\omega^{2}\right)^{2}+d_{0}^{2} \omega^{2}\right]^{2}}, \\
& \frac{d \varphi(x)}{d x}=\frac{4 R^{2} C_{0}}{\pi^{3}} \omega \int_{0}^{\infty} \frac{\left(\Omega^{2}-\omega^{2}\right) l N(l) d l}{\left[\left(\Omega^{2}-\omega^{2}\right)^{2}+d_{0}^{2} \omega^{2}\right]} \\
& \quad-2 \mu P \varepsilon_{m}^{m}\left|\cos K_{p} x\right|^{m} \Omega_{p}^{q} d_{0}^{2} \omega^{3} \\
& \quad \times \int_{0}^{\infty} \frac{\left(\Omega^{2}-\omega^{2}\right) l N(l) d l}{\left[\left(\Omega^{2}-\Omega_{p}^{2}\right)^{2}+d_{0}^{2} \Omega_{p}^{2}\right]^{m / 2}\left[\left(\Omega^{2}-\omega^{2}\right)^{2}+d_{0}^{2} \omega^{2}\right]^{2}} \\
& \quad+\eta Q \varepsilon_{m}^{n}\left|\cos K_{p} x\right|^{n} \Omega_{p}^{r} \omega \\
& \quad \times \int_{0}^{\infty} \frac{\left[\left(\Omega^{2}-\omega^{2}\right)^{2}-d_{0}^{2} \omega^{2}\right] \Omega^{2} l N(l) d l}{\left[\left(\Omega^{2}-\Omega_{p}^{2}\right)^{2}+d_{0}^{2} \Omega_{p}^{2}\right]^{n / 2}\left[\left(\Omega^{2}-\omega^{2}\right)^{2}+d_{0}^{2} \omega^{2}\right]^{2}}, \\
& \quad
\end{aligned}
$$

где

$$
\begin{aligned}
P= & \frac{8 R^{2} C_{0}}{\pi^{9 / 2}} \frac{(1+q) \Gamma[(m+3) / 2]}{\Gamma[(m+4) / 2]} B\left[\frac{m-q+1}{2}, \frac{q+1}{2}\right] \\
& \times\left(\frac{4 R C_{0}^{2}}{\pi^{2} b^{2}}\right)^{m}\left(\frac{b}{C_{\perp}}\right)^{q}, \\
Q= & \frac{8 R^{2} C_{0}}{\pi^{9 / 2}} \frac{(1+n-r) \Gamma[(n+3) / 2]}{\Gamma[(n+4) / 2]} B\left[\frac{n-r+1}{2}, \frac{r+1}{2}\right] \\
& \times\left(\frac{4 R C_{0}^{2}}{\pi^{2} b^{2}}\right)^{n}\left(\frac{b}{C_{\perp}}\right)^{r},
\end{aligned}
$$

$B(m, n)=\frac{\Gamma(m) \Gamma(n)}{\Gamma(m+b)}-$ бета-функция, а функция $N=N(l)$ определяет распределение дислокаций по длинам $l$ после отрыва их сегментов от примесных атомов (под действием мощной НЧ волны накачки), т. е. функция $N=N(l)$ определятся не примесными атомами, а дислокационной сеткой.

Из уравнений (19), (20) находим выражения для нелинейных коэффициента затухания $\chi\left(\varepsilon_{m}, \Omega_{p}, \omega\right)=$ $=\ln \left[a\left(\varepsilon_{m}=0, x=L\right) / a\left(\varepsilon_{m}, x=L\right)\right]$ и фазовой задержки несущей $\tau\left(\varepsilon_{m}, \Omega_{p}, \omega\right)=\left[\varphi\left(\varepsilon_{m}, x=L\right)-\varphi\left(\varepsilon_{m}=0\right.\right.$, $x=L)] / \omega$ для слабой ВЧ волны под действием мощной НЧ волны накачки:

$$
\begin{aligned}
& \chi\left(\varepsilon_{m}, \Omega_{p}, \omega\right)=\frac{\mu P}{\sqrt{\pi}} \frac{\Gamma[(m+1) / 2]}{\Gamma[(m+2) / 2]} \varepsilon_{m}^{m} L \Omega_{p}^{q} d_{0} \omega^{2} \\
& \times \int_{0}^{\infty} \frac{\left[\left(\Omega^{2}-\omega^{2}\right)^{2}-d_{0}^{2} \omega^{2}\right] l N(l) d l}{\left[\left(\Omega^{2}-\Omega_{p}^{2}\right)^{2}+d_{0}^{2} \Omega_{p}^{2}\right]^{m / 2}\left[\left(\Omega^{2}-\omega^{2}\right)^{2}+d_{0}^{2} \omega^{2}\right]^{2}} \\
& +\frac{2 \eta Q}{\sqrt{\pi}} \frac{\Gamma[(n+1) / 2]}{\Gamma[(n+2) / 2]} \varepsilon_{m}^{n} L \Omega_{p}^{r} d_{0} \omega^{2} \\
& \times \int_{0}^{\infty} \frac{\left(\Omega^{2}-\omega^{2}\right) \Omega^{2} l N(l) d l}{\left[\left(\Omega^{2}-\Omega_{p}^{2}\right)^{2}+d_{0}^{2} \Omega_{p}^{2}\right]^{n / 2}\left[\left(\Omega^{2}-\omega^{2}\right)^{2}+d_{0}^{2} \omega^{2}\right]^{2}}, \\
& \tau\left(\varepsilon_{m}, \Omega_{p}, \omega\right)=-\frac{2 \mu P}{\sqrt{\pi}} \frac{\Gamma[(m+1) / 2]}{\Gamma[(m+2) / 2]} \varepsilon_{m}^{m} L \Omega_{p}^{q} d_{0}^{2} \omega^{2} \\
& \times \int_{0}^{\infty} \frac{\left(\Omega^{2}-\omega^{2}\right) l N(l) d l}{\left[\left(\Omega^{2}-\Omega_{p}^{2}\right)^{2}+d_{0}^{2} \Omega_{p}^{2}\right]^{m / 2}\left[\left(\Omega^{2}-\omega^{2}\right)^{2}+d_{0}^{2} \omega^{2}\right]^{2}} \\
& +\frac{\eta Q}{\sqrt{\pi}} \frac{\Gamma[(n+1) / 2]}{\Gamma[(n+2) / 2]} \varepsilon_{m}^{n} L \Omega_{p}^{r} \\
& \times \int_{0}^{\infty} \frac{\left[\left(\Omega^{2}-\omega^{2}\right)^{2}-d_{0}^{2} \omega^{2}\right] \Omega^{2} l N(l) d l}{\left[\left(\Omega^{2}-\Omega_{p}^{2}\right)^{2}+d_{0}^{2} \Omega_{p}^{2}\right]^{n / 2}\left[\left(\Omega^{2}-\omega^{2}\right)^{2}+d_{0}^{2} \omega^{2}\right]^{2}} .
\end{aligned}
$$

Здесь вследствие динамического характера НЧ волны накачки, а также из-за того, что $q \geq 0$ и $r \geq 0$, выражения для $\chi\left(\varepsilon_{m}, \Omega_{p}, \omega\right)$ и $\tau\left(\varepsilon_{m}, \Omega_{p}, \omega\right)$ имеют несколько более сложный вид по сравнению 
с аналогичными выражениями (15), (16), когда нагрузка - статическая $\left(\varepsilon_{0}=\right.$ const $)$ и $q, r=0$ (в НЧ приближении $\left(\omega \ll \Omega, \Omega^{2} / d_{0}\right)$ выражения $(21),(22)$ существенно упрощаются). Из анализа амплитудно-частотных зависимостей выражений для $\chi\left(\varepsilon_{m}, \Omega_{p}, \omega\right)$ и $\tau\left(\varepsilon_{m}, \Omega_{p}, \omega\right)$ от $\varepsilon_{m}, \Omega_{p}$ и $\omega$ и сравнения их с результатами соответствующих экспериментов можно определить показатели степени и коэффициенты диссипативной и реактивной нелинейности дислокаций, параметр демпфирования, распределение дислокаций по длинам и их плотность. В НЧ диапазоне $\left(\omega \ll \Omega, \Omega^{2} / d_{0}\right)$ при $m<n$ каждая из этих нелинейностей отвечает только за „свой“ эффект: диссипативная - за затухание звука на звуке, а реактивная - за фазовую задержку несущей. Из выражений (21), (22) следует, что в этом случае, при одинаковых длинах дислокаций $(N(l)=$ $\left.=\left(\Lambda / l_{0}\right) \delta\left(l-l_{0}\right), \quad \Omega_{0}=[2 /(1-v)]^{1 / 2}\left(C_{\perp} / l_{0}\right)\right), \quad$ знаки нелинейных коэффициента затухания и фазовой задержки несущей ВЧ волны зависят от частоты $\omega$ : в диапазонах $0<\omega<\Omega_{1}=\left[-d_{0}+\left[d_{0}^{2}+4 \Omega_{0}^{2}\right]^{1 / 2}\right] / 2$ и $\omega>\Omega_{2}=\left[d_{0}+\left[d_{0}^{2}+4 \Omega_{0}^{2}\right]^{1 / 2}\right] / 2-\chi_{n l}\left(\varepsilon_{m}, \Omega_{p}, \omega\right)>0$, $\tau\left(\varepsilon_{m}, \Omega_{p}, \omega\right)>0, \quad$ а в диапазоне $\Omega_{1}<\omega<\Omega_{2} \quad-$ $\chi_{n l}\left(\varepsilon_{m}, \Omega_{p}, \omega\right)<0, \tau\left(\varepsilon_{m}, \Omega_{p}, \omega\right)<0$, т. е. в поликристаллах, вообще говоря, может наблюдаться не только затухание, но и „усиление“ звука на звуке. Из (21), (22) также видно, что на низких частотах $\left(\omega \ll \Omega_{0}, \Omega_{0}^{2} / d_{0}\right) \quad \chi_{n l}\left(\varepsilon_{m}, \Omega_{p}, \omega\right) \propto \omega^{2}$, а на высоких $\left(\omega \gg \Omega_{2}\right)-\chi_{n l}\left(\varepsilon_{m}, \Omega_{p}, \omega\right) \propto \omega^{-2}$. Следовательно, в диапазоне промежуточных частот могут наблюдаться „промежуточные “ зависимости $\chi_{n l}\left(\varepsilon_{m}, \Omega_{p}, \omega\right)$ от $\omega ;$ их конкретный вид определяется параметром демпфирования и функцией распределения дислокаций.

В заключение этого раздела отметим, что кроме рассмотренных „усредненных“ (т.е. не зависящих от времени и фазы $\theta$ НЧ волны) эффектов изменения амплитуды и фазы несущей слабой ВЧ волны под действием мощной НЧ волны накачки, в таком резонаторе возможны эффекты амплитудной и фазовой модуляции первичных и генерации вторичных ВЧ волн, в первую очередь - на комбинационных частотах $\omega_{ \pm}(2 z)=\omega \pm 2 z \Omega_{p}, z=1,2,3, \ldots$, однако вследствие эффекта затухания звука на звуке амплитуда этих вторичных ВЧ волн будет незначительной. Тем не менее при взаимодействии амплитудно-модулированной мощной НЧ волны накачки и слабой гармонической ВЧ волны в таких нелинейных средах будут наблюдаться эффекты амплитудно-фазовой модуляции (АФМ) слабой ВЧ волны, связанные с переносом на нее амплитудной модуляции мощной НЧ волны накачки [34,35]. В этом случае параметры диссипативной и реактивной нелинейности поликристалла можно определить на основе анализа АФМ модуляции ВЧ волны (или амплитудных зависимостей ее спектральных составляющих).

\section{4. Амплитудно-фазовые эфффекты самовоздействия интенсивной высокочастотной волны}

Рассмотрим амплитудно-фазовые эффекты самовоздействия интенсивной гармонической ВЧ волны, приводящие к зависимости коэффициента затухания и фазовой скорости волны от ее амплитуды. (Такие эффекты наблюдались в стержнях из свинца [25], цинка [22], гранита [29], магнезита [27], мрамора [30] и кварцита [28]). В этом случае в отличие от эффектов влияния мощной НЧ на распространение слабой ВЧ волн функция распределения сегментов дислокаций $N=N(l)$ будет определяться примесными атомами, а не дислокационной сеткой. Это связано с тем, что для ВЧ волн отрыва дислокаций от примесных атомов не происходит, поэтому их длины сегментов дислокаций определяются примесными атомами.

Решение уравнения (11) будем искать методом возмущений, полагая, что $\varepsilon(x, t)=\varepsilon_{1}(x, t)+\varepsilon_{3}(x, t)$, $\left|\varepsilon_{3}(x, t)\right| \ll\left|\varepsilon_{1}(x, t)\right|, \quad \varepsilon_{1}(x, t)=a(x) \cos [\omega t-k x-\varphi(x)]$, $\varepsilon_{1}(x=0, t)=a_{0} \cos \omega t, \quad \varepsilon_{3}(x=0, t)=0, \quad d a(x) / d x \ll k a(x)$, $d \varphi(x) / d x \ll k \varphi(x)$. После несложных вычислений получаем уравнения для амплитуды $a(x)$ и фазы $\varphi(x)$ волны $\varepsilon_{1}(x, t)$ :

$$
\begin{aligned}
& \frac{1}{a(x)} \frac{d a(x)}{d x}=-\frac{4 R^{2} C_{0}}{\pi^{3}} Z(\omega)-2 \mu H(m, q) a^{m}(x) d_{0} \omega^{q+2} \\
& \times \int_{0}^{\infty} \frac{\left[\left(\Omega^{2}-\omega^{2}\right)^{2}-d_{0}^{2} \omega^{2}\right] l N(l) d l}{\left[\left(\Omega^{2}-\omega^{2}\right)^{2}+d_{0}^{2} \omega^{2}\right]^{\frac{m}{2}+2}}-4 \eta F(n, r) a^{n}(x) d_{0} \omega^{r+2} \\
& \times \int_{0}^{\infty} \frac{\left(\Omega^{2}-\omega^{2}\right) \Omega^{2} l N(l) d l}{\left[\left(\Omega^{2}-\omega^{2}\right)^{2}+d_{0}^{2} \omega^{2}\right]^{\frac{n}{2}+2}}, \\
& \frac{d \varphi(x)}{d x}=\frac{4 R^{2} C_{0}}{\pi^{3}} W(\omega)-4 \mu H(m, q) a^{m}(x) d_{0}^{2} \omega^{q+3} \\
& \times \int_{0}^{\infty} \frac{\left(\Omega^{2}-\omega^{2}\right) l N(l) d l}{\left[\left(\Omega^{2}-\omega^{2}\right)^{2}+d_{0}^{2} \omega^{2}\right]^{\frac{m}{2}+2}}+2 \eta F(n, r) a^{n}(x) \omega^{r+1} \\
& \times \int_{0}^{\infty} \frac{\left[\left(\Omega^{2}-\omega^{2}\right)^{2}-d_{0}^{2} \omega^{2}\right] \Omega^{2} l N(l) d l}{\left[\left(\Omega^{2}-\omega^{2}\right)^{2}+d_{0}^{2} \omega^{2}\right]^{\frac{n}{2}+2}},
\end{aligned}
$$

где

$$
\begin{aligned}
H(m, q)= & \frac{8 R^{2} C_{0}}{\pi^{9 / 2}} \frac{\Gamma[(m+3) / 2]}{\Gamma[(m+4) / 2]} B\left[\frac{m-q+1}{2}, \frac{q+3}{2}\right] \\
& \times\left(\frac{4 R C_{0}^{2}}{\pi^{2} b^{2}}\right)^{m}\left(\frac{b}{C_{\perp}}\right)^{q}, \\
F(n, r)= & \frac{8 R^{2} C_{0}}{\pi^{9 / 2}} \frac{\Gamma[(n+3) / 2]}{\Gamma[(n+4) / 2]} B\left[\frac{n-r+3}{2}, \frac{r+1}{2}\right] \\
& \times\left(\frac{4 R C_{0}^{2}}{\pi^{2} b^{2}}\right)^{n}\left(\frac{b}{C_{\perp}}\right)^{r} .
\end{aligned}
$$


Первые слагаемые в правых частях уравнений (23), (24) отвечают за линейные затухание и изменение скорости распространения ВЧ волны, а вторые и третьи - описывают изменения ее амплитуды и фазы (т.е. фазовой скорости волны) за счет соответственно диссипативной и реактивной нелинейности. В относительно низкочастотном диапазоне и при $m<n$ каждая из этих нелинейностей отвечает также только за „свой“ эффект: диссипативная - за нелинейное ограничение амплитуды волны (или самопросветление среды), а реактивная за изменение фазовой задержки несущей (т. е. фазовой скорости волны):

$$
\begin{aligned}
\frac{1}{a(x)} \frac{d a(x)}{d x} & =-\frac{4 R^{2} C_{0}}{\pi^{3}} Z(\omega)-2 \mu H(m, q) a^{m}(x) d_{0} \omega^{q+2} \\
& \times \int_{0}^{\infty} \frac{\left[\left(\Omega^{2}-\omega^{2}\right)^{2}-d_{0}^{2} \omega^{2}\right] l N(l) d l}{\left[\left(\Omega^{2}-\omega^{2}\right)^{2}+d_{0}^{2} \omega^{2}\right]^{\frac{m}{2}+2}} \\
\frac{d \varphi(x)}{d x}= & \frac{4 R^{2} C_{0}}{\pi^{3}} W(\omega)+2 \eta F(n, r) a^{n}(x) \omega^{r+1} \\
& \times \int_{0}^{\infty} \frac{\left[\left(\Omega^{2}-\omega^{2}\right)^{2}-d_{0}^{2} \omega^{2}\right] \Omega^{2} l N(l) d l}{\left[\left(\Omega^{2}-\omega^{2}\right)^{2}+d_{0}^{2} \omega^{2}\right]^{\frac{n}{2}+2}}
\end{aligned}
$$

Из уравнений (25), (26) находим выражения для амплитуды $a(L)$ и нелинейной задержки несущей $\tau_{1}\left(a_{0}, \omega\right)=\left[\varphi\left(a_{0}, x=L\right)-\varphi(0, x+L)\right] / \omega$ волны $\varepsilon_{1}(x, t)$ при $x=L: \tau_{1}\left(a_{0}, \omega\right)=\left[\varphi\left(a_{0}, x=L\right)-\varphi(0, x=L)\right] / \omega$

$$
\begin{gathered}
a(L)=\frac{a_{0} \exp \left[-A_{1}(\omega) L\right]}{\left[1+\frac{B_{1}(\omega)}{A_{1}(\omega)}\left\{1-\exp \left[-m A_{1}(\omega) L\right]\right\} a_{0}^{m}\right]^{\frac{1}{m}}}, \\
\tau_{1}\left(a_{0}, \omega\right)=B_{2}(\omega) \int_{0}^{L} a^{n}(x) d x,
\end{gathered}
$$

где

$$
\begin{gathered}
A_{1}(\omega)=\frac{4 R^{2} C_{0}}{\pi^{3}} Z(\omega), \\
B_{1}(\omega)=2 \mu H(m, q) d_{0} \omega^{q+2} \int_{0}^{\infty} \frac{\left[\left(\Omega^{2}-\omega^{2}\right)^{2}-d_{0}^{2} \omega^{2}\right] l N(l) d l}{\left[\left(\Omega^{2}-\omega^{2}\right)^{2}+d_{0}^{2} \omega^{2}\right]^{\frac{m}{2}+2}}, \\
B_{2}(\omega)=2 \eta F(n, r) \omega^{r} \int_{0}^{\infty} \frac{\left[\left(\Omega^{2}-\omega^{2}\right)^{2}-d_{0}^{2} \omega^{2}\right] \Omega^{2} l N(l) d l}{\left[\left(\Omega^{2}-\omega^{2}\right)^{2}+d_{0}^{2} \omega^{2}\right]^{\frac{n}{2}+2}} .
\end{gathered}
$$

На малых расстояниях, при $m A_{1}(\omega) L \ll 1, n A_{1}(\omega) L \ll 1$, $m B_{1}(\omega) L a_{0}^{m} \ll 1, \quad$ получаем: $a(L) \approx a_{0} \exp \left[-A_{1}(\omega) L\right]$, $\tau_{1}\left(a_{0}, \omega\right) \approx B_{2}(\omega) a_{0}^{n} L$.

При $\frac{B_{1}(\omega)}{A_{1}(\omega)}\left\{1-\exp \left[-m A_{1}(\omega) L\right]\right\} a_{0}^{m} \ll 1 \quad$ из выражений (27) имеем

$$
\begin{aligned}
a(L) \cong & a_{0} \exp \left[-A_{1}(\omega) L\right]\left[1-\frac{B_{1}(\omega)}{m A_{1}(\omega)}\right. \\
& \left.\times\left\{1-\exp \left[-m A_{1}(\omega) L\right]\right\} a_{0}^{m}\right],
\end{aligned}
$$

$$
\begin{aligned}
& \tau_{1}\left(a_{0}, \omega\right) \cong a_{0}^{n} \frac{\left.1-\exp \left[-n A_{1} \omega\right) L\right]}{n A_{1}(\omega)}\left[1-\frac{n B_{1}(\omega)}{m A_{1}(\omega)}\right. \\
& \left.\times\left(1-\frac{n}{n+m} \frac{1-\exp \left[-(n+m) A_{1}(\omega) L\right]}{1-\exp \left[-n A_{1}(\omega) L\right]}\right) a_{0}^{m}\right] B_{2}(\omega),
\end{aligned}
$$

a при выполнении дополнительных условий $\exp \left[-m A_{1}(\omega) L\right] \ll 1, \exp \left[-n A_{1}(\omega) L\right] \ll 1-$ получаем

$$
\begin{gathered}
a(L) \cong a_{0} \exp \left[-A_{1}(\omega) L\right]\left[1-\frac{B_{1}(\omega)}{m A_{1}(\omega)} a_{0}^{m}\right], \\
\tau_{1}\left(a_{0}, \omega\right) \cong \frac{B_{2}(\omega) a_{0}^{n}}{n A_{1}(\omega)}\left[1-\frac{n B_{1}(\omega)}{(n+m) A_{1}(\omega)} a_{0}^{m}\right] .
\end{gathered}
$$

Из выражений (27)-(29) следует, что при одинаковых длинах сегментов дислокаций $[N(l)=$ $\left.=\left(\Lambda / l_{0}\right) \delta\left(l-l_{0}\right)\right], \quad \Omega=[2 /(1-v)]^{1 / 2}\left(C_{\perp} / l_{0}\right) \quad$ знаки коэффициентов $B_{1,2}(\omega)$, а определяющих нелинейные коэффициенты затухания и фазовой задержки несущей ВЧ волны, зависят от ее частоты $\omega$. В диапазонах $0<\omega<\Omega_{1}=\left\{-d_{0}+\left[d_{0}^{2}+4 \Omega^{2}\right]^{1 / 2}\right\} / 2$ и $\omega>\Omega_{2}=\left\{d_{0}+\left[d_{0}^{2}+4 \Omega^{2}\right]^{1 / 2}\right\} / 2-B_{1,2}(\omega)>0$, поэтому здесь будет наблюдаться эффект ограничения амплитуды волны, при этом $\tau_{1}\left(a_{0}, \omega\right)>0$. В диапазоне $\Omega_{1}<\omega<\Omega_{2}-B_{1,2}(\omega)<0$, так что здесь будет иметь место эффект самопросветления среды, при этом $\tau_{1}\left(a_{0}, \omega\right)<0$.

\section{5. Генерация третьей гармоники интенсивной высокочастотной волны}

Кроме эффектов самовоздействия интенсивной первичной гармонической волны, в поликристаллах возможна и генерация вторичных волн на частотах высших гармоник, что также можно использовать для изучения их дислокационной акустической нелинейности. Генерация второй и третьей гармоник в металлах исследовалась в работах [12,36-38], где учитывались квадратичная решеточная нелинейность однородного твердого тела [31] и кубичная упругая нелинейность, связанная с изменением длины дислокационной струны при ее изгибе под действием статического и переменного напряжений. Здесь в рамках волнового уравнения (8) будет рассмотрен процесс генерации третьей гармоники при распространении интенсивной продольной ВЧ волны в поликристалле с диссипативной и реактивной дислокационной нелинейностью. Из-за нечетного характера диссипативной и реактивной нелинейности уравнения движения дислокаций при нулевой статической нагрузке $\sigma_{0}=0$ в поликристалле будут генерироваться только нечетные гармоники.

Подставляя в уравнение $(11) \varepsilon(x, t)=\varepsilon_{1}(x, t)+\varepsilon_{3}(x, t)$, $\varepsilon_{1}(x, t)=a(x) \cos [\omega t-k x-\varphi(x)], \quad \varepsilon_{3}(x, t)=b_{3}(x) \sin \{3[\omega t$ $-k x-\varphi(x)]\}+c_{3}(x) \cos \{3[\omega t-k x-\varphi(x)]\}$ и полагая, что $b_{3}(x) \ll a(x), \quad c_{3}(x) \ll a(x), \quad d b_{3}(x) d x \ll 3 k b_{3}(x)$, $d c_{3}(x) d x \ll 3 k c_{3}(x)$, получаем уравнения для амплитуд 
$b_{3}(x)$ и $c_{3}(x)$ sin- и cos-компонент вторичной волны $\varepsilon_{3}(x, t)$ :

$$
\begin{aligned}
& \frac{d b_{3}(x)}{d x}-3 c_{3}(x) \frac{d \varphi(x)}{d x}=-\frac{4 R^{2} C_{0}}{\pi^{3}}\left[b_{3}(x) Z(3 \omega)\right. \\
& \left.+c_{3}(x) W(3 \omega)\right]-\frac{24 R^{2} C_{0}}{\pi^{4}} \delta d_{0} a^{m+1}(x) \omega^{q}\left(\frac{4 R C_{0}^{2}}{\pi^{2} b}\right)^{m} \\
& \times\left(\frac{3 m-4 q}{m+4}\right) B\left[\frac{m-q+1}{2}, \frac{q+3}{2}\right] X_{m}(3 \omega) \\
& -\frac{24 R^{2} C_{0}}{\pi^{4}} g a^{n+1}(x) \omega^{r}\left(\frac{4 R C_{0}^{2}}{\pi^{2} b}\right)^{n}\left(\frac{n-4 r}{n+4}\right) \\
& \times B\left[\frac{n-r+3}{2}, \frac{r+1}{2}\right] \bar{Y}_{n}(3 \omega), \\
& \frac{d c_{3}(x)}{d x}+3 b_{3}(x) \frac{d \varphi(x)}{d x}=\frac{4 R^{2} C_{0}}{\pi^{3}}\left[b_{3}(x) W(3 \omega)\right. \\
& \left.-c_{3}(x) Z(3 \omega)\right]-\frac{24 R^{2} C_{0}}{\pi^{4}} \delta d_{0} a^{m+1}(x) \omega^{q}\left(\frac{4 R C_{0}^{2}}{\pi^{2} b}\right)^{m} \\
& \times\left(\frac{3 m-4 q}{m+4}\right) B\left[\frac{m-q+1}{2}, \frac{q+3}{2}\right] Y_{m}(3 \omega) \\
& +\frac{24 R^{2} C_{0}}{\pi^{4}} g a^{n+1}(x) \omega^{r}\left(\frac{4 R C_{0}^{2}}{\pi^{2} b}\right)^{n}\left(\frac{n-4 r}{n+4}\right) \\
& \times B\left[\frac{n-r+3}{2}, \frac{r+1}{2}\right] \bar{X}_{n}(3 \omega),
\end{aligned}
$$

где

$$
\begin{gathered}
Z(3 \omega)=9 d_{0} \omega^{2} \int_{0}^{\infty} \frac{l N(l) d l}{\left(\Omega^{2}-9 \omega^{2}\right)^{2}+9\left(d_{0} \omega\right)^{2}}, \\
W(3 \omega)=3 \omega \int_{0}^{\infty} \frac{\left(\Omega^{2}-9 \omega^{2}\right) l N(l) d l}{\left(\Omega^{2}-9 \omega^{2}\right)^{2}+9\left(d_{0} \omega\right)^{2}},
\end{gathered}
$$

$X_{m}(3 \omega)=$

$\int_{0}^{\infty} \frac{3 d_{0} \omega^{3} l N(l) d l}{\left[\left(\Omega^{2}-\omega^{2}\right)^{2}+\left(d_{0} \omega\right)^{2}\right]^{(m+1) / 2}\left[\left(\Omega^{2}-9 \omega^{2}\right)^{2}+9\left(d_{0} \omega\right)^{2}\right]}$,

$Y_{m}(3 \omega)=$

$\int_{0}^{\infty} \frac{\omega^{2}\left(\Omega^{2}-9 \omega^{2}\right) l N(l) d l}{\left[\left(\Omega^{2}-\omega^{2}\right)^{2}+\left(d_{0} \omega\right)^{2}\right]^{(m+1) / 2}\left[\left(\Omega^{2}-9 \omega^{2}\right)^{2}+9\left(d_{0} \omega\right)^{2}\right]}$,

$\bar{X}_{n}(3 \omega)=$

$\int_{0}^{\infty} \frac{3 d_{0} \omega^{2} \Omega^{2} l N(l) d l}{\left[\left(\Omega^{2}-\omega^{2}\right)^{2}+\left(d_{0} \omega\right)^{2}\right]^{(n+1) / 2}\left[\left(\Omega^{2}-9 \omega^{2}\right)^{2}+9\left(d_{0} \omega\right)^{2}\right]}$,
$\bar{Y}_{n}(3 \omega)=$

$\int_{0}^{\infty} \frac{\omega\left(\Omega^{2}-9 \omega^{2}\right) \Omega^{2} l N(l) d l}{\left[\left(\Omega^{2}-\omega^{2}\right)^{2}+\left(d_{0} \omega\right)^{2}\right]^{(n+1) / 2}\left[\left(\Omega^{2}-9 \omega^{2}\right)^{2}+9\left(d_{0} \omega\right)^{2}\right]}$,

a амплитуда $a(x)$ и фаза $\varphi(x)$ первичной волны определяются уравнениями $(27)-(29)$. Из (30), (31) получаем уравнение для комплексной амплитуды $e_{3}(x)=b_{3}(x)+j c_{3}(x)$ вторичной волны $\varepsilon_{3}(x, t)$ :

$$
\frac{d e_{3}(x)}{d x}+3[\beta(3 \omega)+j \Psi(3 \omega)] e_{3}(x)=s_{3}(x)
$$

где

$$
\begin{gathered}
\beta(3 \omega)=\frac{4 R^{2} C_{0}}{3 \pi^{3}} Z(3 \omega), \\
\Psi(3 \omega)=\frac{d \varphi(x)}{d x}-\frac{4 R^{2} C_{0}}{3 \pi^{3}} W(3 \omega) \approx \frac{4 R^{2} C_{0}}{\pi^{3}} \\
\times\left[W(\omega)-\frac{W(3 \omega)}{3}\right], \\
s_{3}(x)=-\frac{24 R^{2} C_{0}}{\pi^{4}} \delta d_{0} a^{m+1}(x) \omega^{q}\left(\frac{4 R C_{0}^{2}}{\pi^{2} b}\right)^{m}\left(\frac{3 m-4 q}{m+4}\right) \\
\times B\left[\frac{m-q+1}{2}, \frac{q+3}{2}\right]\left[X_{m}(3 \omega)+j Y_{m}(3 \omega)\right] \\
+j \frac{24 R^{2} C_{0}}{\pi^{4}} g a^{n+1}(x) \omega^{r}\left(\frac{4 R C_{0}^{2}}{\pi^{2} b}\right)^{n}\left(\frac{n-4 r}{n+4}\right) \\
\times B\left[\frac{n-r+3}{2}, \frac{r+1}{2}\right]\left[\bar{X}_{n}(3 \omega)+j \bar{Y}_{n}(3 \omega)\right] .
\end{gathered}
$$

Решение уравнения (32) имеет вид

$$
e_{3}(x)=C_{3}(x) \exp \left[\frac{4 R^{2} C_{0}}{\pi^{3}}[Z(3 \omega)+j W(3 \omega)] x-3 j \varphi(x)\right],
$$

где

$$
\begin{aligned}
& C_{3}(x)=\frac{24 R^{2} C_{0}}{\pi^{4}} \delta d_{0} \omega^{q}\left(\frac{4 R C_{0}^{2}}{\pi^{2} b}\right)^{m}\left(\frac{3 m-4 q}{m+4}\right) \\
& \times B\left[\frac{m-q+1}{2}, \frac{q+3}{2}\right]\left[X_{m}(3 \omega)+j Y_{m}(3 \omega)\right] \int_{0}^{x} a^{m+1}\left(x_{1}\right) \\
& \times \exp \left[-\frac{4 R^{2} C_{0}}{\pi^{3}}[Z(3 \omega)+j W(3 \omega)] x_{1}+3 j \varphi\left(x_{1}\right)\right] d x_{1} \\
& +j \frac{24 R^{2} C_{0}}{\pi^{4}} g \omega^{r}\left(\frac{4 R C_{0}^{2}}{\pi^{2} b}\right)^{n}\left(\frac{n-4 r}{n+4}\right) B\left[\frac{n-r+3}{2}, \frac{r+1}{2}\right] \\
& \left.\times\left[\bar{X}_{n}(3 \omega)+j \bar{Y}_{n}(3 \omega)\right]\right\} \int_{0}^{x} a^{n+1}\left(x_{1}\right) \exp \left[-\frac{4 R^{2} C_{0}}{\pi^{3}}\right. \\
& \left.\times[Z(3 \omega)+j W(3 \omega)] x_{1}+3 j \varphi\left(x_{1}\right)\right] d x_{1} .
\end{aligned}
$$


Вообще говоря, решение (33) является достаточно сложным для анализа; комплексная амплитуда $e_{3}(x)$ волны $\varepsilon_{3}(x, t)$ определяется суперпозицией волн, генерируемых за счет реактивной и диссипативной нелинейностей, при этом $a=a\left(a_{0}, x\right)$. В связи с этим мы рассмотрим поведение амплитуды деформации $e_{3}$ на малых расстояниях $x$, когда эффекты самовоздействия для первичной волны $\varepsilon_{1}(x, t)$ почти не проявляются и $a(x) \approx a_{0} \exp \left[-A_{1}(\omega) x\right]$, $\varphi(x) \approx \frac{4 R^{2} C_{0}}{\pi^{3}} W(\omega) x:$

$$
\begin{aligned}
& C_{3}(x)=\frac{24 R^{2} C_{0}}{\pi^{4}} \delta d_{0} \omega^{q}\left(\frac{4 R C_{0}^{2}}{\pi^{2} b}\right)^{m}\left(\frac{3 m-4 q}{m+4}\right) B\left[\frac{m-q+1}{2}, \frac{q+3}{2}\right]\left[X_{m}(3 \omega)+j Y_{m}(3 \omega)\right] \\
& \times a_{0}^{m+1} \frac{1-\exp \left(-\frac{4 R^{2} C_{0}}{\pi^{3}}\{(m+1) Z(\omega)-Z(3 \omega)-j[3 W(\omega)-j W(3 \omega)]\} x\right)}{\frac{4 R^{2} C_{0}}{\pi^{3}}\{(m+1) Z(\omega)-Z(3 \omega)-j[3 W(\omega)-j W(3 \omega)]\}} \\
& +j \frac{24 R^{2} C_{0}}{\pi^{4}} g \omega^{r}\left(\frac{4 R C_{0}^{2}}{\pi^{2} b}\right)^{n}\left(\frac{n-4 r}{n+4}\right) B\left[\frac{n-r+3}{2}, \frac{r+1}{2}\right]\left[\bar{X}_{n}(3 \omega)+j \bar{Y}_{n}(3 \omega)\right] \\
& \times a_{0}^{n+1} \frac{1-\exp \left(-\frac{4 R^{2} C_{0}}{\pi^{3}}\{(n+1) Z(\omega)-Z(3 \omega)-j[3 W(\omega)-W(3 \omega)]\} x\right)}{\frac{4 R^{2} C_{0}}{\pi^{3}}\{(n+1) Z(\omega)-Z(3 \omega)-j[3 W(\omega)-W(3 \omega)]\}} .
\end{aligned}
$$

Приведем оценки для параметров $\mu$ и $\eta$ диссипативной и реактивной нелинейностей для изотропного поликристалла при $m=n=1, q=r=0$, $b=3 \cdot 10^{-10} \mathrm{~m}, \quad N(l)=\left(\Lambda / l_{0}\right) \delta\left(l-l_{0}\right), \quad \Lambda=10^{11} \mathrm{~m}^{-2}$, $l_{0}=10^{-5} \mathrm{~m}, \quad C_{\perp}=2.2 \cdot 10^{3} \mathrm{~m} / \mathrm{s}, \quad C_{0}=3.7 \cdot 10^{3} \mathrm{~m} / \mathrm{s}$, $d_{0}=10^{8} \mathrm{rad} / \mathrm{s}, \quad v=0.3, \quad \Omega \approx 3.9 \cdot 10^{8} \mathrm{rad} / \mathrm{s} . \quad$ Полагая, что при распространении в таком поликристалле первичной волны с частотой $\omega=2 \pi f, f=10 \mathrm{MHz}$ и начальной амплитудой деформации $a_{0}=10^{-5}$, волна на частоте третьей гармоники генерируется только на диссипативной $(\eta=0)$ или только на реактивной $(\mu=0)$ нелинейности и на расстоянии $L=10 \mathrm{~cm}$ еe амплитуда составляет $\left|e_{3}(L)\right|=10^{-8}$, получим соответственно $\mu \cong 63$ и $\eta \cong 4.8$.

\section{Заключение}

В работе на основе модификации линейной ВЧ части дислокационной теории Гранато-Люкке получено волновое уравнение для поликристаллических твердых тел с дислокационной диссипативной и реактивной акустической нелинейностью. Проведены теоретические исследования нелинейных эффектов влияния статического напряжения на распространение слабой продольной упругой волны, взаимодействия НЧ и ВЧ продольных упругих волн, а также самовоздействия интенсивной ВЧ продольной волны и генерации ее третьей гармоники. Получены выражения для:

- изменений коэффициента затухания и фазовой задержки несущей слабой ВЧ волны под действием статического напряжения и мощной НЧ волны накачки;

- амплитуды и фазовой задержки несущей интенсивной гармонической волны;

— амплитуды третьей гармоники интенсивной гармонической волны.
Описанные нелинейные эффекты (затухание и фазовая задержка несущей слабой ВЧ волны под действием сильной НЧ волны и самовоздействие интенсивной ВЧ волны) наблюдались в экспериментах со многими поликристаллическими горными породами и некоторыми металлами [20-30], при этом в каждом таком материале эти эффекты являются чрезвычайно сильными и, как правило, проявляют различные амплитудно-частотные зависимости. Так, например, в отожжженной поликристаллической меди (в зависимости от температуры отжига) уменьшение амплитуды ВЧ волны под действием мощной НЧ волны накачки $\left(\varepsilon_{m} \approx 10^{-5}\right)$ составляет от 2 до 10 (и даже более) раз, а относительная фазовая задержка несущей достигает величины $1 \%$. Следует, однако, заметить, что подобные значения нелинейных затухания и фазовой задержки несущей слабой ВЧ волны (при прочих равных условиях) наблюдаются далеко не во всех материалах, а в некоторых из них (например, в силикатном и органическом стеклах, неотожженной меди, дюралюминии, стали, молибдене, никеле, олове, титане и т.д.) такие эффекты не наблюдаются вообще. Кроме того, если ту же отожженную медь подвергнуть пластической деформации изгиба или кручения, то интенсивность нелинейных эффектов в этом металле сильно уменьшается. Все это свидетельствует о том, что диссипативная и реактивная нелинейность является чувствительной структурной характеристикой многих поликристаллических твердых тел, что можно использовать для акустической диагностики их дислокационной структуры.

\section{Финансирование работы}

Работа поддержана РФФИ (грант N20-02-00215A). 


\section{Конфликт интересов}

Автор заявляет, что у него нет конфликта интересов.

\section{Список литературы}

[1] H.G. Van Bueren. Imperfections in crystals. (Interscience, NY., 1960).

[2] J. Friedel. Dislocations. (Pergamon Press, Oxford-London-Edinburgh-New York-Paris-Frankfurt, 1964).

[3] D. Hull. Introduction to Dislocations. (Pergamon Press, Oxford-London-Edinburgh-New York-Paris-Frankfurt, 1964).

[4] R.W.K. Honeycombe. The Plastic Deformation of Metals. (Edward Arnold (Publishers) Ltd, 1968).

[5] A.S. Nowick, B.S. Berry. Anelastic Relaxation in Crystalline Solids. (Academic Press, New York and London, 1972).

[6] J.P. Hirth, J. Lothe. Theory of Dislocations. (McGraw Hill, 1970).

[7] A. Granato, K. Lucke. J.Appl.Phys., 27 (5), 583 (1956).

[8] Ультразвуковые методы исследования дислокаций: Сб. статей / Пер. с англ. и нем. под ред. Л.Г. Меркулова. (ИИЛ, М., 1963).

[9] „Application to quantum and solid state physics", in Physical Acoustics: Principles and Methods, Edited by Warren P. Mason (Academic Press, New York and London, Vol. 4, Part A, 1966).

[10] „The effect of imperfection“, in Physical Acoustics: Principles and Methods, Edited by Warren P. Mason (Academic Press, New York and London, Vol. 3, Part A, 1966).

[11] Д. Ниблетт, Дж. Уилкс. УФН, 80 (1), 125 (1963).

[12] R. Truell, C. Elbaum. B.B. Chick. Ultrasonic Methods in Solid State Physics. (Academic Press, New York and London, 1969).

[13] T. Suzuki. Dislocation Dynamics. Ed. Rosenfeld A.R. et al. (McGraw Hill, NY., 1967/68).

[14] H.F. Pollard. Sound Waves in Solids. (Pion Limited, 1977).

[15] J.S. Koehler. In book: Imperfection in Nearly Perfect Crystals, (NY., 1952).

[16] V.E. Nazarov, A.V. Radostin. Nonlinear acoustic waves in micro-inhomogeneous solids. (John Wiley \& Sons, 2015).

[17] A.S. Novick. Phys. Rev., 80 (2), 249 (1950).

[18] S. Takahachi. J. Phys. Soc. Japan. 11 (12), 1253 (1956).

[19] D.N. Beshers. J. Appl. Phys., 30 (2), 252 (1959).

[20] В.Е. Назаров. Акуст. журн., 37 (4), 825 (1991).

[21] В.Е. Назаров. Акуст. журн., 37 (6), 1177 (1991).

[22] V.E. Nazarov. Acoust.Lett., 15 (2), 22 (1991).

[23] С.В. Зименков, В.Е. Назаров. ФММ, 73 (3), 62 (1992).

[24] V.E. Nazarov, A.B. Kolpakov. JASA, 107 (4), 1915 (2000).

[25] В.Е. Назаров. ФММ, 88 (4), 82 (1999).

[26] С.В. Зименков, В.Е. Назаров. Изв. РАН, Сер. Физика Земли, 1, 13 (1933).

[27] V.E. Nazarov, A.B. Kolpakov. Ultrasonics, 54, 471 (2014).

[28] V.E. Nazarov, A.B. Kolpakov. Wave motion, 72, 187 (2017).

[29] V.E. Nazarov, A.B. Kolpakov, A.V. Radostin. Acoust. Phys., 56 (4), 453 (2010).

[30] В.Е. Назаров, А.Б. Колпаков, А.В. Радостин. Физическая мезомеханика, 13 (2), 41 (2010).

[31] Л.Д. Ландау, Е.М. Лифшиц. Теория упругости. (Наука, М., 1986).
[32] Э. Камке. Справочник по обыкновенным дифберенциальным уравнениям. Пер. с нем. С.В. Фомина. (Наука, M., 1976).

[33] В.Е. Назаров. Акуст. журн., 57 (2), 204 (2011).

[34] А.Л. Багмет, В.Е. Назаров, А.В. Николаев, А.П. Резниченко, А.М. Поликарпов. ДАН, 346 (3), 390 (1996).

[35] В.Е. Назаров, А.В. Радостин. Акуст. журн., 57 (5), 596 (2011).

[36] T. Suzuki, A. Hikata, C. Elbaum. J. Appl. Phys., 35 (9), 2761 (1964).

[37] A. Hikata, B.B. Chick, C. Elbaum. J. Appl. Phys., 36 (1), 229 (1965).

[38] A. Hikata, C. Elbaum. Phys. Rev., 144 (2), 469 (1966). 